\title{
Ordering of the three-dimensional Heisenberg spin glass in magnetic fields
}

\author{
Hikaru Kawamura* and Daisuke Imagawa \\ Department of Earth and Space Science, Faculty of Science, Osaka University, Toyonaka 560-0031, Japan
}

(November 4, 2018)

\begin{abstract}
Spin and chirality orderings of the three-dimensional Heisenberg spin glass are studied under magnetic fields in light of the recently developed spin-chirality decoupling-recoupling scenario. It is found by Monte Carlo simulations that the chiral-glass transition and the chiral-glass ordered state, which are essentially of the same character as their zero-field counterparts, occur under magnetic fields. Implication to experimental phase diagram is discussed.
\end{abstract}

In the studies of spin glasses, much effort has been devoted to the properties under magnetic fields. Unfortunately, our understanding of them still has remained unsatisfactory [1]. Most of the numerical studies have focused on the properties of the simple Ising model, especially the three-dimensional (3D) Edwards-Anderson (EA) model. While the existence of a true thermodynamic spin-glass (SG) transition has been established for this model in zero field, the question of its existence or nonexistence in magnetic fields has remained unsettled.

If one tries to understand real experimental SG ordering, one has to remember that many of real SG materials are more or less Heisenberg-like rather than Ising, in the sense that the random magnetic anisotropy is considerably weaker than the isotropic exchange interaction [1,2. Numerical simulations have indicated that the isotropic 3D Heisenberg SG with finite-range interaction does not exhibit the conventional SG order at finite temperature in zero field 值5. Since applied fields generally tend to suppress the SG ordering, a true thermodynamic SG transition is even more unlikely under magnetic fields in case of the Heisenberg model.

Experimentally, however, a rather sharp transition-like behavior has been observed under magnetic fields in typical Heisenberg-like SG magnets, e.g., canonical SG like $\mathrm{AuFe}$ and $\mathrm{CuMn}$, although it is not completely clear whether the observed anomaly corresponds to a true thermodynamic transition [1, 6, ,6]. The situation is in contrast to the zero-field case where the existence of a true thermodynamic SG transition has been established experimentally [1]. Set aside the question of the strict nature of the SG "transition", it is experimentally observed that a weak applied field lowers the zero-field SG transition temperature rather quickly [1,6,7]. For higher fields, the SG "transition" becomes much more robust to fields, where the "transition temperature" shows much less field dependence [1,6, 67. Such behaviors of the SG transition temperature under magnetic fields $T_{g}(H)$ were often interpreted in terms of the mean-field model [1.6.6. Indeed, the mean-field Sherrington-Kirkpatrick (SK) model with an infinite-range Heisenberg exchange interaction with weak random magnetic anisotropy ex- hibits a transition line similar to the experimental one 87: i.e., the so-called de Almeida-Thouless (AT) line $H \propto\left(T_{g}(0)-T_{g}(H)\right)^{3 / 2}$ in weak-field regime where the anisotropy is important, and the Gabay-Toulouse (GT) line $H \propto\left(T_{g}(0)-T_{g}(H)\right)^{1 / 2}$ in strong-field regime where the anisotropy is unimportant. Nevertheless, if one notes that the true finite-temperature transition under magnetic fields, though possible in the infinite-range SK model, is unlikely to occur in a more realistic finite-range Heisenberg model, an apparent success of the meanfield model in explaining the experimental phase diagram should be taken with strong reservation.

Recently, one of the present authors has proposed a scenario, the spin-chirality decoupling-recoupling scenario, aimed at explaining some of the puzzles concerning the experimentally observed SG transition in zero field [3]. In this scenario, chirality, which is a multispin variable representing the sense or the handedness of local noncoplanar spin structures induced by spin frustration, plays an essential role. In a fully isotropic Heisenberg $\mathrm{SG}$, in particular, this scenario claims the occurrence of a novel chiral-glass ordered state in which only the chirality exhibits a glassy long-range order (LRO) while the spin remains paramagnetic. At the chiral-glass transition, among the global symmetries of the Hamiltonian, only the $Z_{2}$ spin reflection (inversion) symmetry is broken spontaneously with keeping the $S O(3)$ spin rotation symmetry preserved. Note that this picture entails the spin-chirality (or $S O(3)-Z_{2}$ ) decoupling on long length and time scales: Namely, although the chirality is not independent of the spin on microscopic length scale, it eventually exhibits a long-distance behavior entirely different from the spin. Such a chiral-glass transition was indeed observed in zero field in a recent Monte Carlo simulation by Hukushima and Kawamura [5]. It was also found there that the critical properties associated with the chiral-glass transition were different from those of the Ising SG, and that the chiral-glass ordered state exhibited a one-step-like novel RSB.

In the chirality scenario of Ref. [3], experimental SG transition in real Heisneberg-like $\mathrm{SG}$ magnets is regarded essentially as a chiral-glass transition "revealed" via the

*E-mail : kawamura@ess.sci.osaka-u.ac.jp 
magnetic anisotropy. Weak but finite random magnetic anisotropy inherent to real magnets "recouples" the spin to the chirality, and the chiral-glass transition shows up as an experimentally observable spin-glass transition.

The purpose of the present Letter is to reexamine the SG ordering of the 3D isotropic Heisenberg SG under applied fields in light of the above chirality scenario. We first argue some of the possible consequences of the chirality picture on the finite-field SG properties, and then perform extensive Monte Carlo (MC) simulations to check how the spin and chirality really order in fields. We show that the chiral-glass phase, essentially of the same character as the zero-field one, remains stable as a true thermodynamic phase in applied fields. It is also found that the associated chiral-glass transition line possesses some of the character of the GT line of the mean-field model, yet its physical origin entirely different.

Though we expect that our argument holds quite generally, we fix here our model Hamiltonian, which is just the one used in our MC simulation below. We consider the isotropic classical Heisenberg model on a 3D simple cubic lattice,

$$
\mathcal{H}=-\sum_{<i j>} J_{i j} \vec{S}_{i} \cdot \vec{S}_{j}-H \sum_{i} S_{i}^{z},
$$

where $H$ is the intensity of the magnetic field applied along the $z$ direction. The nearest-neighbor coupling $J_{i j}$ is assumed to take the value $J$ or $-J$ with equal probability ( $\pm J$ distribution). Local scalar chirality is defined for three neighboring spins: Here we define it at the $i$-th site and in the $\mu$-th direction $(\mu=x, y, z)$ by $\chi_{i \mu}=\vec{S}_{i-\hat{e}_{\mu}} \cdot \vec{S}_{i} \times \vec{S}_{i+\hat{e}_{\mu}}, \hat{e}_{\mu}$ being a unit lattice vector in the $\mu$-th direction.

Applied fields reduce the global symmetry of the Hamiltonian (1) from the zero-field one $Z_{2} \times S O(3)$ to $Z_{2} \times S O(2)$, where $Z_{2}$ refers to the spin reflection with respect to an arbitrary plane including the field axis, and $S O(2)$ to the spin rotation around the field axis. Note that, even in fields, the chiral $Z_{2}$ reflection symmetry, characterized by the mutually opposite signs of the chirality, is kept intact. The chirality is a pseudoscalar invariant under $S O(2)$ (or $S O(3)$ in case of $H=0$ ) rotations but changes sign under $Z_{2}$ reflections. The same chiral $Z_{2} \times S O(2)$ symmetry also appears in the $X Y \mathrm{SG}$ with two-component spins [9].

Since the chiral $Z_{2}$ is supposed to be decoupled from the spin $S O(3)$ already in zero field, and the applied field serves only to reduce the decoupled $S O(3)$ to $S O(2)$, one naturally expects that the $Z_{2}$ chiral-glass transition, essentially of the same type as the zero-field one, would persist even under magnetic fields 10. More specifically, the chiral-glass transition in a field should lie in the same universality class as the zero-field one, characterized by the same set of exponents, and the chiral-glass ordered state in a field exhibits the same kind of one-step-like RSB as in zero field.

Since the chiral-glass order in a field is expected to be essentially of the same character as in zero field, the chiral-glass transition temperature under magnetic fields $T_{\mathrm{CG}}(H)$ should be a regular function of $H$. If one takes account of the obvious symmetry $H \leftrightarrow-H$, the chiralglass transition line at low enough fields should behave as

$$
T_{\mathrm{CG}}(0)-T_{\mathrm{CG}}(H)=c H^{2}+c^{\prime} H^{4}+\cdots \text {. }
$$

Generally, the coefficient $c$ could be either positive or negative. Interestingly, the above form of the chiral transition line is similar to the so-called GT line of the meanfield Heisenberg SK model. We emphasize, however, that their physical origin is entirely different. The quadratic dependence of the chiral-glass transition line is simply of regular origin, whereas that of the GT-line in the SK model cannot be regarded so.

Concerning the spin order, applied fields trivially induce a nonzero longitudinal (parallel to the field) spin order at any temperature. The behavior of the transverse (perpendicular to the field) component could be more nontrivial. If one recalls the recent numerical results on the 3D $X Y$ SG which indicate the absence of the standard SG order at least just below the chiral-glass transition [9, 11], the transverse spin order is also unlikely to arise in the present case, at least just below the $Z_{2}$ chiral-glass transition.

In order to examine whether the above expectation really holds or not, we next perform extensive MC simulations on the isotropic $\pm J$ Heisenberg SG model (1). Simulations are performed for a variety of fields $H / J=$ $0.05,0.1,0.5,2.0,3.0,5.0$. The lattices studied are simplecubic lattices with $L^{3}$ sites with $L=6 \sim 16$ with periodic boundary conditions. The system is fully equilibrated with use of the temperature-exchange method [13. We show here the data explicitly for the particular field value of $H / J=0.5$, where the sample average is taken over 128-800 bond realizations.

By running two independent sequences of systems (replica 1 and 2) in parallel, we compute a scalar chiral overlap $q_{\chi}$ between the chiralities of the two replicas by $q_{\chi}=\frac{1}{3 N} \sum_{i \mu} \chi_{i \mu}^{(1)} \chi_{i \mu}^{(2)}$, as well as a spin-overlap tensor $q_{\mu \nu}$ between the transverse $\mu$ and $\nu$ components $(\mu$, $\nu=x, y)$ of the spin by $q_{\mu \nu}=\frac{1}{N} \sum_{i} S_{i \mu}^{(1)} S_{i \nu}^{(2)}$. Then, in terms of these overlaps, we calculate the Binder ratios of the chirality $g_{\chi}$, and of the transverse $(X Y)$ components of the spin $g_{T}$ defined in the standard manner: See Ref. [5] for detailed definition. The results are shown in Fig.1. The Binder ratio of the chirality $g_{\chi}$ exhibits a negative dip which, with increasing $L$, tends to deepen and shift toward lower temperature. Furthermore, $g_{\chi}$ of various $L$ cross at a temperature slightly above the dip temperature $T_{\text {dip }}$ on negative side of $g_{\chi}$, eventually merging at temperatures lower than $T_{\text {dip }}$. The observed behavior of $g_{\chi}$ is similar to the one observed in zero field [5]. As argued in Ref. [5], the persistence of a negative dip and the crossing occurring at $g_{\chi}<0$, are strongly suggestive of the occurrence of a finite-temperature transition where $g_{\chi}\left(T_{\mathrm{CG}}^{-}\right)$ 
and $g_{\chi}\left(T_{\mathrm{CG}}\right)$ take negative values in the $L \rightarrow \infty$ limit. In the inset of Fig.1(a), we plot the negative-dip temperature $T_{\text {dip }}(L)$ versus $1 / L$. The data lie on a straight line fairly well, and its extrapolation to $1 / L=0$ gives an estimate of the bulk chiral-glass transition temperature, $T_{\mathrm{CG}} / J \sim 0.25$. (More precisely, $T_{\mathrm{dip}}(L)$ should scale with $L^{1 / \nu}$ where $\nu$ is the chiral-glass correlation-length exponent. As shown below, our estimate of $\nu \simeq 1.3$ comes close to unity, more or less justifying the linear extrapolation employed here. Extrapolation with respect to $L^{1 / 1.3}$ yields $T_{\mathrm{CG}} / J \sim 0.23$.)

In sharp contrast to $g_{\chi}$, Binder ratio of the transverse component of the spin $g_{T}$ decreases monotonically toward zero with increasing $L$, without a negative dip nor a crossing, suggesting that the transverse component of spin remains disordered even below $T_{\mathrm{CG}}$.

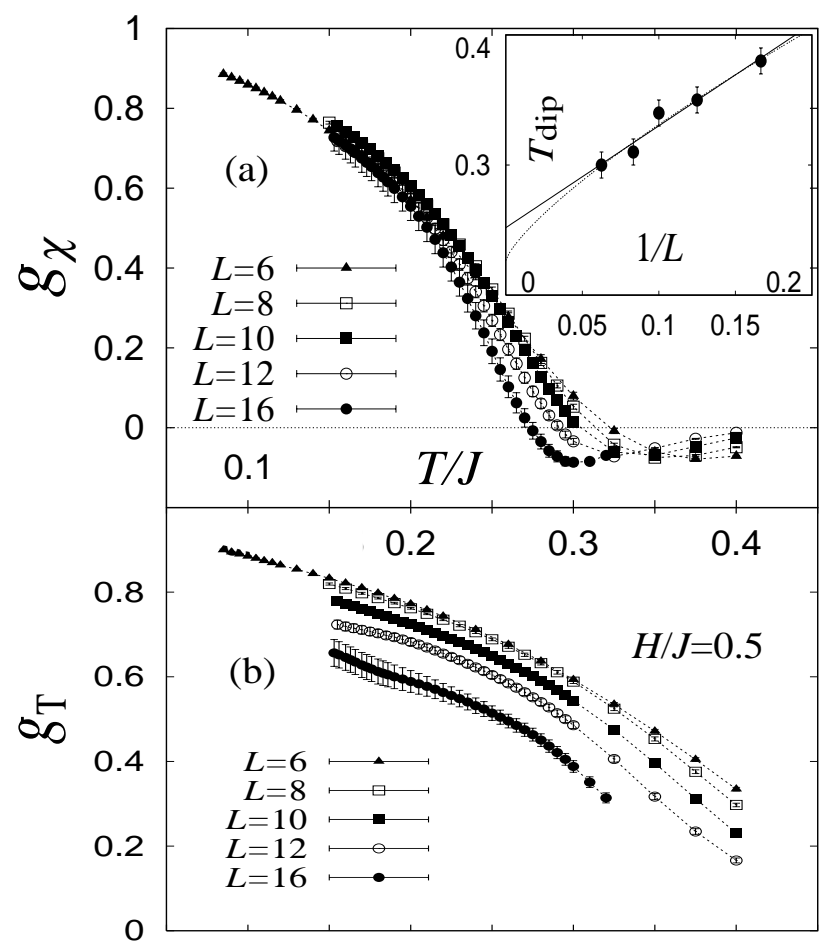

FIG. 1. The temperature and size dependence of the Binder ratios of the chirality (a), and of the transverse $(X Y)$ component of the spin (b), in a field $H / J=0.5$. Inset displays the negative-dip temperature vs. $1 / L$. The solid and broken lines are the best fits assuming the $1 / L$ and $1 / L^{1 / 1.3}$ dependence, respectively.

Validity of our estiamte of $T_{\mathrm{CG}}$ has been checked also from the behavior of other quantities, e.g., the temporaldecay of the equilibrium chirality autocorrelation function (data not shown here), which yields $T_{\mathrm{CG}} / J=$ $0.23(2)$ in accord with the above estimate. In order to probe the possible RSB in the chiral-glass ordered state, we display in Fig.2 the distribution function of the chiraloverlap defined by $P\left(q_{\chi}^{\prime}\right)=\left[\left\langle\delta\left(q_{\chi}-q_{\chi}^{\prime}\right)\right\rangle\right]$ where $\langle\cdots\rangle$ and $[\cdots]$ represent the thermal average and the sample average, respectively. The existence of a growing "central peak" at $q_{\chi}=0$ for larger $L$, in addition to the standard "side-peaks" corresponding to $\pm q_{\mathrm{CG}}^{\mathrm{EA}}$, suggests the occurrence of a one-step-like peculiar RSB in the chiralglass ordered state. Similar behavior was observed in the chiral-glass state in zero field [5]. The existence of a persistent negative dip in the Binder ratio $g_{\chi}$ is also consistent with the occurrence of such a one-step-like RSB [12.

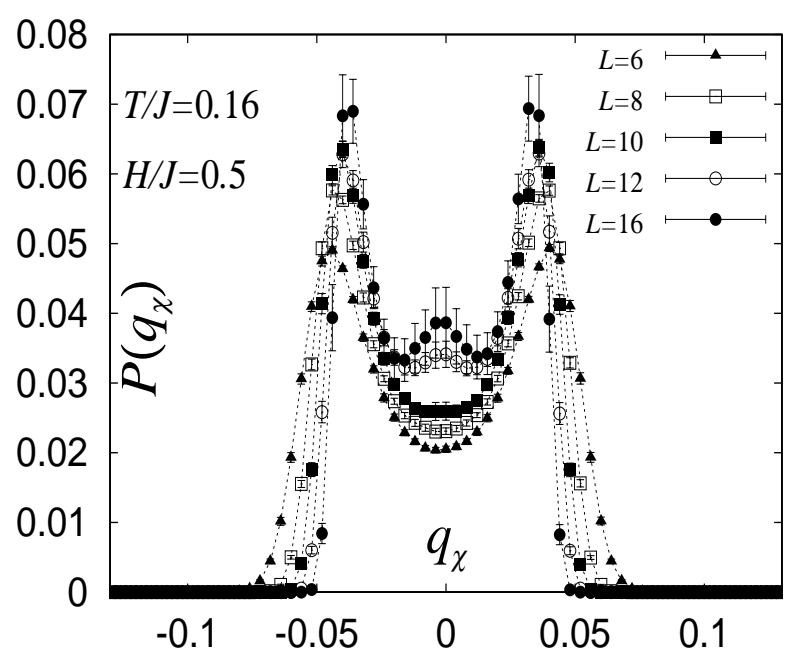

FIG. 2. Chiral-overlap distribution function at $T / J=0.16$, well below the chiral-glass transition point $T_{\mathrm{CG}} / J \simeq 0.23$, in a field of $H / J=0.5$.

With setting $T_{\mathrm{CG}} / J=0.23$ as determined above, we perform the standard finite-size scaling of the chiral-glass order parameter $\left.\left[<q_{\chi}^{2}\right\rangle\right]$ and of the chiral autocorrelation function, to estimate various chiral-glass exponents. We then get $\nu=1.3(2), \eta=0.6(3), z=5.3(5)$, which turns out to agree within errors with the corresonding zero-field exponents of Ref. [5]. The results seem consistent with a common universality class occurring both in zero-field and finite-field chiral-glass transitions.

Similar calculations and analysis are repeated for other field values as well. For $H / J=0.1$, in particular, we have performed the same scale of intensive calculation as was done for $H / J=0.5$, to find that all qualitative features are similar. The chiral-glass transition with a one-steplike RSB occurs at $T_{\mathrm{CG}} / J=0.21(2)$, with the exponents $\nu=1.3(2), \eta=0.6(3), z=4.9(5)$, which agrees within errors with our estimates for $H / J=0.5$.

By collecting the $T_{\mathrm{CG}}$ values determined for other field values, we construct a phase diagram in the temperaturemagnetic field plane as shown in Fig.3. The chiral-glass state remains quite robust against magnetic fields. Indeed, $T_{\mathrm{CG}}(H)$ is not much reduced from the zero-field value even at field as large as ten times of $T_{\mathrm{CG}}(0)$. This somewhat surprising property probably arises from the 
fact that the magnetic field couples in the Hamiltonian directly to the spin, not to the chirality, and the effective coupling between the field and the chirality is rather weak. At lower fields, the chiral-glass transition line is almost orthogonal to the $H=0$ axis, consistent with the expected behavior Eq.(2). Our data are even not inconsistent with the coefficient $c$ in Eq.(2) being slightly negative so that $T_{\mathrm{CG}}(H)$ initially increases slightly with $H$, though it is difficult to draw a definite conclusion due to the scatter of our estimate of $T_{\mathrm{CG}}(H)$.

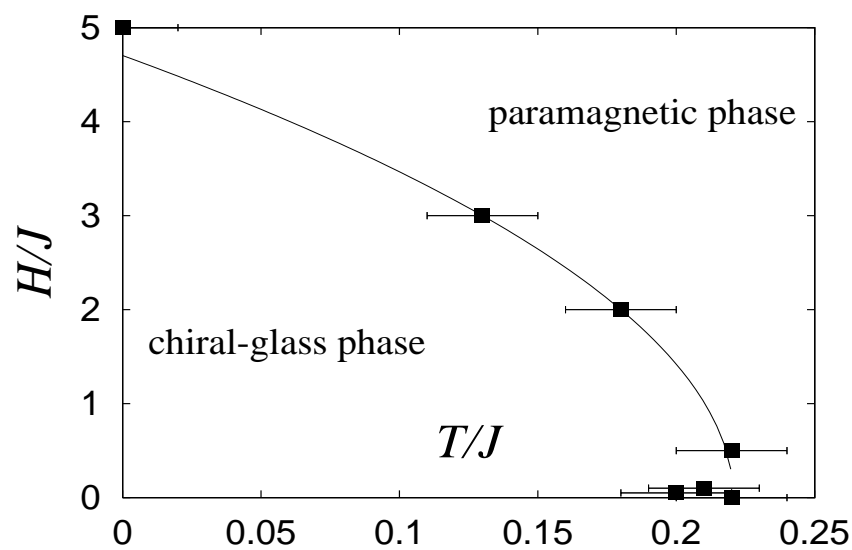

FIG. 3. The temperature - magnetic field phase diagram of the $3 \mathrm{D}$ isotropic $\pm J$ Heisenberg spin glass. Note the difference in energy scales of the temperature- and the field-axes.

Finally, we wish to discuss implications of our results to real experimental SG. In real Heisenberg-like SG magnets, weak but finite random magnetic anisotropy neglected here plays a role. Among other things, the anisotropy recouples the spin to the chirality, transforming the chiral-glass state into the spin-glass state. Furthermore, in the presence of both random magnetic anisotropy and magnetic field, all global symmetries of the Hamiltonian will be lost. Nevertheless, we expect that the chiral-glass transition should still persist in fields as a pure RSB transition, not accompanying the global $Z_{2}$-symmetry breaking. Difference in the broken symmetries in zero- and in finite fields, however, causes a singular crossover behavior in fields, in sharp contrast to the fully isotropic case. We believe that this singular crossover line expected in the weak-field regime of anisotropic system is nothing but the AT-like transition line ubitiously observed experimentally. Further detailed nature of this crossover expected in the low-field regime will be discussed in a separate paper [14].

Meanwhile, in the strong-field regime where the applied field dominates the anisotropy, main features of the experimental phase diagram would be described by our present results on isotropic system. Thus, the experimental observation of the GT-like field-insensitive transition line in the high-field regime is fully consistent with our observation of the field-insensitive chiral-glass transition line [1],6.7]. Other interesting possibility revealed by our analysis is that the SG transition line might extend to higher fields than hitherto suspected. For example, for canonical SG AuFe, Campbell et al recently determined the SG phase boundary in fields by torque measurements [7]: At lower fields, applied fields rapidly suppress the SG transition giving rise to the standard AT-like behavior, whereas around the maximal fields of the measurements $(\sim 7 \mathrm{~T})$ the SG transition line becomes almost-field independent. If one roughly estimates the effective $J$ of this system from its zero-field SG transition temperature, it is of order $50 \mathrm{~K}$. If one assumes that the energy scale of our present model calculation could roughly be applicable to $\mathrm{AuFe}$, the SG ordered state should extend to fields much higher than $10 \mathrm{~T}$ without much reduction, or even with a slight increase, in $T_{g}$, although, considering the difference in microscopic details between the present model and real $\mathrm{AuFe}$, one cannot expect a truely quantitative correspondence. Anyway, further high-field experiments on AuFe and on other Heisenberg-like SG magnets might be worthwhile to determine the SG phase boundary in the high-field regime.

The numerical calculation was performed on the Hitachi SR8000 at the supercomputer center, ISSP, University of Tokyo. The authors are thankful to Dr.K. Hukushima for useful discussion.

[1] For reviews on spin glasses, see e. g., (a) K. Binder and A. P. Young, Rev. Mod. Phys. 58, 801 (1986); (b) K. H. Fischer and J. A. Hertz, Spin Glasses Cambridge University Press (1991); (c) J. A. Mydosh, Spin Glasses Taylor \& Francis (1993); (d) A. P. Young (ed.), Spin glasses and random fields World Scientific, Singapore (1997).

[2] J. A. Olive, A. P. Young and D. Sherrington, Phys. Rev. B34, 6341 (1986).

[3] H. Kawamura, Phys. Rev. Lett. 68, 3785 (1992); Int. Jour. Mod. Phys. 7, 345 (1996).

[4] H. Kawamura, Phys. Rev. Lett. 80, 5421 (1998).

[5] K. Hukushima and H. Kawamura, Phys. Rev. E61, R1008 (2000).

[6] D. Chu, G.G. Kennig and R. Orbach, Phys. Rev. Lett. 72, 3270 (1994).

[7] D. Petit, L. Fruchter and I.A. Campbell, Phys. Rev. Lett. 83, 5130 (1999).

[8] G. Kotliar and H. Sompolinsky,, Phys. Rev. Lett 53, 1751 (1984).

[9] H. Kawamura, Phys. Rev. B 51, 12398 (1995); H. Kawamura and M.S. Li, unpublished.

[10] One can consider here a more complicated possibility that the change of the symmetry $S O(3) \rightarrow S O(2)$ affects the $Z_{2}$ chiral part in a nontrivial way, say, seriously modifies the nature of the chirality-chirality interaction, and might lead to the finite-field chiral transition qual- 
itatively different from the zero-field one. However, we restrict ourselves here to the simplest possibility neglecting such possible complications.

[11] J. Maucourt and D. R. Grempel, Phys. Rev. Lett. 80, 770 (1998).
[12] K. Hukushima and H. Kawamura, Phys. Rev. E62, 3360 (2000).

[13] K. Hukushima and K. Nemoto, J. Phys. Soc. Jpn. 65, 1604 (1996).

[14] H. Kawamura, unpublished. 